\title{
Prevalence of Partial Edentulism According to Kennedy's Classification in Wardha Population as per Gender, Age and Their Perception towards Replacement of Teeth
}

\author{
Shreya Makkasare ${ }^{1}$, Seema Sathe Kambala², Tanvi Jaiswal ${ }^{3}$ \\ ${ }^{1}$ SPDC, Sawangi (Meghe), Wardha, Maharashtra, India. ${ }^{2}$ Department of Prosthodontics, SPDC, \\ Sawangi (Meghe), Wardha, Maharashtra, India. ${ }^{3}$ Department of Prosthodontics, SPDC, Sawangi \\ (Meghe), Wardha, Maharashtra, India.
}

\section{ABSTRACT}

\section{BACKGROUND}

Dental and Oral Health is a vital part of our overall health and well-being. Poor oral hygiene can lead to caries and gingival diseases. Maintaining healthy teeth and gums is a lifelong commitment. However, many older adults experience poor oral health. Even though recent reports have shown a consistent decline in the prevalence of tooth loss during the past few decades, significant variation in tooth loss distribution remains. We wanted to determine the prevalence of partial edentulism according to Kennedy's classification and assess the reason for non-replacement of teeth.

\section{METHODS}

This cross-sectional study was conducted among 100 patients who came to the Department of Prosthodontics in SPDC. They were partially edentulous patients in the age group of 20-60 years. After obtaining informed consent from these patients, thorough intraoral examination of all the patients was carried out to identify the pattern \& type of edentulism. Each patient was asked to fill a self-administered questionnaire regarding loss of teeth. The questionnaire comprised of a total of 10 close ended questions. Patient responses were assessed, and analysed.

\section{RESULTS}

Health status was assessed in different age groups and significant results were found. Lack of awareness was found to be the main reason for not replacing the missing teeth in $36 \%$ of patients in the age group of $20-30.36 \%$ patients of the age group 30 40 felt no difference after extraction showing that lack of awareness in young age group remains the main reason for not getting treatment done. Self-motivation resulted in seeking treatment for $40 \%$ patients in the age group of 40-50 whereas it was $60 \%$ in the age group of 20-30 in females; motivation by friends and relatives for seeking treatment affected $50 \%$ of male patients in the age group of 20-30 and same was seen in females of the age group 50-60. 31\% patients in the age group of 20-30 consulted dentist for the replacement of the missing teeth.

\section{CONCLUSIONS}

Gender comparison in this study shows prevalence of loss of teeth with predominant Kennedy's Class 1 in males than females but with less tendency to seek treatment for the same than females.

\section{KEY WORDS}

Kennedy's Classification, Missing Tooth, Gender, Age, Perception, Replacement of Teeth
Corresponding Author:

Dr. Seema Sathe Kambala,

Professor and HOD,

Department of Prosthodontics,

SPDC, Sawangi (Mehge), Wardha-442001,

Maharashtra, India.

E-mail: seemasathe2011@gmail.com

DOI: 10.14260/jemds/2020/161

Financial or Other Competing Interests: None.

How to Cite This Article:

Makkasare S, Kambala SS, Jaiswal T, Prevalence of partial edentulism according to Kennedy's classification in Wardha population as per gender, age and their perception towards replacement of teeth. J. Evolution Med. Dent. Sci. 2020;9(10):741 744, DOI: 10.14260/jemds/2020/161

Submission 20-12-2019,

Peer Review 08-02-2020,

Acceptance 14-02-2020,

Published 09-03-2020. 


\section{BACKGROUND}

Partial edentulousness in dental arch occurs when one or more but not all natural teeth are lost. The loss of many teeth usually affects the quality of life and has a negative impact on the functional, social and psychological well-being of an individual. ${ }^{1}$ Generally, it occurs by caries, periodontal problems, traumatic injuries, impactions, and supernumerary teeth, neoplastic and cystic lesions. Some studies have reported caries as the main causative agent for tooth loss. ${ }^{2}$ The variation in number and location of the edentulous space and its relation to remaining natural teeth necessitates to classify the partial edentulous arches. The main disadvantage of RPD is the damage that is caused to the adjacent tissues and remaining natural teeth. ${ }^{3}$

Associated with this is accumulation of plaque, caries and resorption of residual ridge supporting the RPD. Simple estimation of the proportion of partially edentulous persons is a rough indication of the prevalence of dental diseases and the success or failure of the dental arch. The purpose of partial edentulous arches classification is that it provides communication between dental colleagues, students, technician about the case and for treatment planning and to design the partial denture. It also predicts the difficulties commonly occurring with particular removable partial denture design. ${ }^{4}$ Kennedy's classification (which was originally proposed by Edward Kennedy in 1925) of partially edentulous arch, is universally acceptable. ${ }^{3}$ Kennedy divided all partially edentulous arches into four basic classes. ${ }^{5}$

- Class I- Bilateral edentulous areas located posterior to the natural teeth.

- Class II- A unilateral edentulous areas located posterior to the remaining natural teeth.

- Class III- A unilateral edentulous areas with natural teeth remaining both anterior and posterior to it.

- Class IV- A single, but bilateral (crossing the midline), edentulous area located anterior to the remaining natural teeth.

Therefore, based on above classification this study was carried out to evaluate the prevalence of Kennedy's classification in Wardha population as per gender, Age and their perception towards replacement of teeth.

\section{METHODS}

This cross-sectional study was conducted on 100 patients $^{5}$ who came to the Department of Prosthodontics in SPDC after the ethical clearance from the Institutional Ethical Committee (DMIMS (DU)/IEC/Jun-2019/8105). They were partially edentulous patients between ages 20-60 years. After obtaining informed consent of these patients, thorough intraoral examination was carried out of all the patients to identify the pattern\& type of edentulism. Each patient was asked to fill selfadministered validated questionnaire from SHPER regarding loss of tooth. The questionnaire comprised of total 10 close ended questions. The patient responses were assessed, and analysis was carried out. Partially edentulous patients between 20-60 years of age were included in the study. Patients with missing $3^{\text {rd }}$ molars were excluded from the study. They were divided into four groups as Group 1: 20-30 years of age, Group 2: 30-40 years of age, Group 3:40-50 years of age, Group 4: 50-60 years of age.

\section{Statistical Analysis}

Statistical analysis was performed by using descriptive and inferential statistics using z-test for single proportion and chi square test, software used in the analysis was SPSS 22.0 version and $\mathrm{p}<0.05$ is considered as level of significance.

\section{RESULTS}

Health status was assessed in different age groups and significant results were found, in which $29 \%$ of patients in the age group of 20-30 (male and female) showed the best health status whereas, lowest was seen in males of age group 50-60 and females in age group 30-40. Assessment of frequency of dental visits for male and female was done and in males it was found that $10 \%$ of patients of the age group 40-50 showed least frequent dental visits whereas the age group of 20-30 had more frequency of visits, significantly same results found for female. Dental hygiene maintenance was assessed by tooth brushing habit and result was found that $40 \%$ of male patients of the age group 40-50 and 40\% females of the age group 2030 displayed good maintenance of dental hygiene In case of frequency of missing teeth, $32 \%$ of the male patients in $40-50$ age group and $30 \%$ of the female patients in age group of 50 60 had most maxillary teeth. Mandibular teeth loss was observed in $38 \%$ of both male and female patients in 20-30 age group (graph $1 \& 2$ ).

Caries was found out to be main reason for the loss of teeth in $34 \%$ of patients of the age group 20-30 whereas the least significant reason for loss of teeth was periodontal condition which was observed in $13 \%$ patients among all age groups of both male and female records (graph $3 \& 4$ ). Duration of edentulism of less than 3 months was seen in $44 \%$ male patients in 40-50 age group and 43\% of patient's in 20-30 age group. Duration of edentulism for more than 6 months was observed in $36 \%$ of patients of the age group 20-30 in both genders. $34 \%$ of the male patients in the age group $20-30$ were previous prosthesis users whereas $38 \%$ females in same age group showed no use of any prosthesis. No use of previous prosthesis was observed in $37 \%$ of male patients in the age group of $40-50$ and $36 \%$ of female patients in the age group of 50-60 showed highest prosthesis acceptance.

Lack of awareness came out to be the main reason for not replacing the missing teeth in $36 \%$ of patients in the age group of $20-30.36 \%$ patients of the age group 30-40 felt no difference after extraction showing that lack of awareness in young age group remains main reason for not getting treatment done. Self-motivation resulted in seeking treatment for $40 \%$ patients in the age group of $40-50$ whereas it was $60 \%$ in age group of 20-30 in females, Friends and relatives affected $50 \%$ of male patients in the age group of 20-30 and same was seen in females of the age group 50-60.31\% patients in the age group of 20-30 got consultation from dentist for the replacement of the missing teeth. 

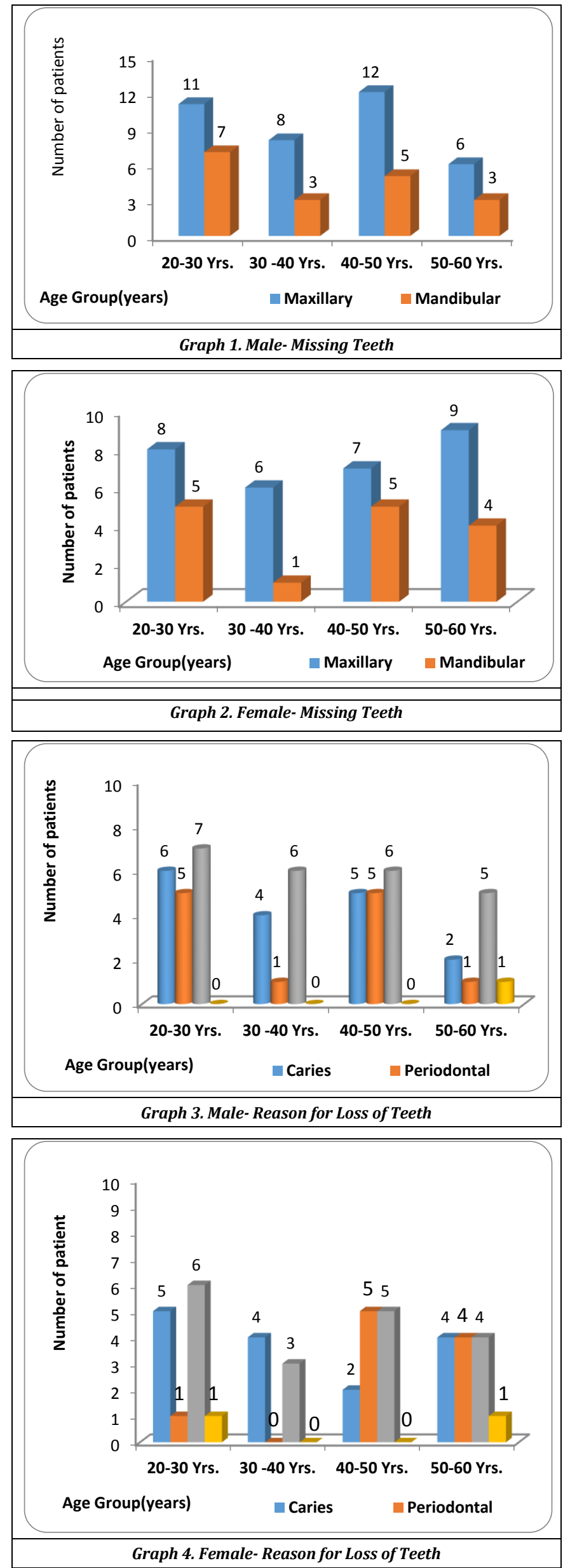

\section{DISCUSSION}

Gender has been an important factor analysed by various authors in prevalence of partial edentulism. Most of the previous studies have shown that there is no significant correlation between gender and occurrence of partial edentulism. But few studies do show a significant difference between gender and various classes of edentulism. In this study, which was conducted in Wardha, there was no gender difference in health status of male and female patients seeking the treatment. There was a gender difference regarding frequency of visits to the dentist. Male patients showed more frequency in 6 months than in females. Dental hygiene in between both genders showed no significant difference. The numbers of missing teeth were seen to be more in males both in maxillary and mandibular arch than in females. Also, it was noticed that Kennedy's class 1 was more prevalent in the patients and was more in males as compared to females. Extraction was the major cause for loss of teeth for males more than females. Duration of edentulism for less than 3 months and more than 6 months showed male predilection. Males showed more tendency than females of using as well as not using the prosthesis. Dentists played a key role in convincing for replacement in majority of patients in both the genders.

Cause of unreplaced missing teeth was found to be lack of awareness in maximum patients and the reason for nonreplacement in male patients was that they found no difference in function even after extraction. They said since they could eat well without teeth, they did not think of replacing them. When a gender comparison was being made about who was better motivated, it was seen that female patients showed better motivation.

Levels of oral health and economic status are unequally distributed throughout the population. ${ }^{6}$ Unlike developed countries, in developing countries like India, individuals with lower income always prefer to fulfil their basic needs first in other words, foods, clothing and shelter; neglecting healthcare. This scenario becomes worst in older people living alone with limited income. This again aids in progression of disease and consequently contributes to worsening of OHRQoL.7

\section{CONCLUSIONS}

Prevalence of loss of teeth with predominant Kennedy's class 1 is seen in males than in females. Tendency to seek treatment for the same is less in males than in females. Males are more prone for edentulism and are more motivated to avail treatment compared to females. 20-30 years age group was the healthiest as they visited the dentist most frequently. On the contrary, 40-50 years age group was the least healthy and less concerned with dental visits and showed maximum maxillary teeth loss. Lack of awareness affects the plan for seeking treatment, but they can be easily motivated by their friends and family. 


\section{REFERENCES}

[1] Almutairy A, Mohn M. Prevalence of partial edentulism among young Saudi women of Qassim and their perception of early tooth loss. International Journal of Dental Research 2017;5:172-6.

[2] Jeyapalan V, Krishnan CS. Partial edentulism and its correlation to age, gender, socio-economic status and incidence of various Kennedy's classes - a literature review. Journal of Clinical and Diagnostic Research 2015;9(6):ZE14-7.

[3] Knezovic-Zlataric D, Clebic A, Valentic-Peruzovic M, et al. The influence of Kennedy's classification, partial denture material and construction on patient's satisfaction. Acta Stomatol Croat 2001;35(1):77-81.
[4] Devishree RA, Sangeetha S, Jain AR. Prevalence of partial edentulism according to Kennedy's classification based on age, gender and arch. Drug Invention Today 2018;10(1):108-10.

[5] Hama AM, Mahmood DK, Abdullah AO. Prevalence and assessment of partially edentulism according to Kennedy's classification in Sulaimani city. Asian Journal of Science and Technology 2016;7(8):3367-70.

[6] Karnam RR, Kumar NS, Eshwar S, et al. Cognitive ability as a determinant of socioeconomic and oral health status among adolescent college students of Bengaluru, India. Journal of Clinical and Diagnostic Research: JCDR 2016;10(12):ZC62-6.

[7] Gondivkar SM, Bhowate RR, Gadbail AR, et al. Impact of socioeconomic inequalities on quality of life in oral submucous fibrosis patients. Future Oncology 2019;15(8):875-83. 